\title{
Set-Membership Fuzzy Filtering for Nonlinear Discrete-Time Systems
}

\author{
Fuwen Yang, Senior Member, IEEE, and Yongmin Li, Senior Member, IEEE
}

\begin{abstract}
This paper is concerned with the set-membership filtering (SMF) problem for discrete-time nonlinear systems. We employ the Takagi-Sugeno (T-S) fuzzy model to approximate the nonlinear systems over the true value of state and to overcome the difficulty with the linearization over a state estimate set rather than a state estimate point in the set-membership framework. Based on the T-S fuzzy model, we develop a new nonlinear SMF estimation method by using the fuzzy modeling approach and the $S$-procedure technique to determine a state estimation ellipsoid that is a set of states compatible with the measurements, the unknown-but-bounded process and measurement noises, and the modeling approximation errors. A recursive algorithm is derived for computing the ellipsoid that guarantees to contain the true state. A smallest possible estimate set is recursively computed by solving the semidefinite programming problem. An illustrative example shows the effectiveness of the proposed method for a class of discrete-time nonlinear systems via fuzzy switch.
\end{abstract}

Index Terms-Convex optimization, linear set-membership filtering (SMF), nonlinear SMF, unknown-but-bounded noise, Takagi-Sugeno (T-S) fuzzy model.

\section{INTRODUCTION}

$\mathbf{T}$ HE filtering problem for nonlinear systems remains challenging and has been attracting considerable research interests over the past four decades. Since the time evolution of the probability density of the state vector conditional on the measurements cannot directly be calculated in most nonlinear cases [2], various approximation methods have been developed in the literature [1], [4], [16], [24], [32], [36]. For nonlinear systems with Gaussian noises, the extended Kalman filtering (EKF) method was used for state estimation, which applied the linear Kalman filtering theory by linearization of the nonlinear systems around the current estimate [16], [24]. However, the EKF may bring large errors in the true posterior mean and covariance and even diverge if the linearization error is not sufficiently small. These drawbacks have been overcome by unscented Kalman filtering (UKF) by using a deterministic

Manuscript received September 17, 2008; revised January 27, 2009 and February 17, 2009. First published July 21, 2009; current version published October 30, 2009. This work was supported in part by the Engineering and Physical Sciences Research Council (EPSRC) of U.K. under Grant $\mathrm{EP} / \mathrm{C} 007654 / 1$ and in part by the National Nature Science Foundation of China under Grant 60874059 and Grant 60604027 . This paper was recommended by Associate Editor H. Gao.

F. Yang is with the School of Information Science and Engineering, East China University of Science and Technology, Shanghai 200237, China. He was with the Department of Information Systems and Computing, Brunel University, UB8 3PH Middlesex, U.K. (e-mail: fwyang @ecust.edu.cn).

$\mathrm{Y}$. Li is with the Department of Information Systems and Computing, Brunel University, UB8 3PH Middlesex, U.K.

Color versions of one or more of the figures in this paper are available online at http://ieeexplore.iee.org.

Digital Object Identifier 10.1109/TSMCB.2009.2020436 sampling approach to capture the mean and covariance estimates with a minimal set of sample points [36]. Recently, a gain-constrained UKF has been developed for nonlinear systems [47]. For nonlinear systems with non-Gaussian noises, a Gaussian sum approach has been proposed for state estimation by density approximation [1]. In this algorithm, the conditional densities are approximated by a sum of Gaussian density functions [32]. An alternative is particle filtering, which is also known as sequential Monte Carlo method [4], which is a sophisticated estimation technique based on simulation. The basic idea of the particle filter is to use a number of independent random variables called particles, which are directly sampled from the state space, to represent the posterior probability and update the posterior by involving the new observations according to the Bayesian rule. However, its computation is very demanding.

The above nonlinear filtering approaches require the system noises, including process noise and measurement noise in a stochastic (Gaussian or non-Gaussian) framework, and then provide a probabilistic state estimation [10], [39]-[41]. The probabilistic nature of the estimates leads to the use of mean and variance to describe the state spreads (distributions). These spreads cannot guarantee that the state is included in some region, because they are not hard bounds. However, in many realworld applications, such as target tracking, system guidance, and navigation, $100 \%$ confidence is required for state estimation. This has motivated the development of an ellipsoidal state estimation. The idea of the ellipsoidal state estimation is to provide a set of state estimates in state space, which always contain the true state of the system by assuming hard bounds on the noise signals (unknown but bounded noises) instead of stochastic descriptions on the system noises [3], [14], [27]. The actual estimate is a set in state space rather than a single vector. These methods are, therefore, known as set-membership or set-valued state estimation (filtering) [3], [27], [38]. We adopt the name set-membership filtering (SMF) in this paper as it is easy to distinguish between a set estimation and a point estimate.

Most publications on SMF deal with linear systems [7], [9], [13], [14], [18], [19], [22], [23], [25], [28]. Only a few consider nonlinear systems [20], [26], as it is not straightforward to use the EKF method where the nonlinear dynamics are linearized around a state estimate point by a first-order Taylor series approximation. In the set-membership framework, linearization should best fit the nonlinear functions over a state estimate set rather than a state estimate point. An approximation method over the entire estimate set has been proposed by minimizing the weighted squared errors between the function values and the 
approximation values, and then an extended SMF has been developed for nonlinear systems by using the linear SMF method [20]. In [26], the nonlinear dynamics are linearized around the current estimate, the remainder terms are then bounded using interval mathematics, and finally the remainder bounds are incorporated as additions to the process or measurement noise bounds. Unfortunately, the above approximations bring a base point error [12] because of the linearization around the estimated value of the state rather than the true value. In this paper, we employ the fuzzy modeling approach to approximate nonlinear systems, because it has been proved that the Takagi-Sugeno (T-S) fuzzy model is a good representation and universal approximator for a certain class of nonlinear dynamic systems [6], [8], [15], [30], [31]. We linearize the nonlinear systems over the true value of state and eliminate the base point error. Based on the T-S fuzzy model [44]-[46], we develop a new nonlinear SMF estimation method. We employ the fuzzy modeling approach and the S-procedure technique [5] to determine a state estimation ellipsoid that is a set of states compatible with the measurements, the unknown-but-bounded process and measurement noises, and the modeling approximation errors. A recursive algorithm is derived for computing the ellipsoid that guarantees to contain the true state. At each step, the ellipsoid is minimized in some sense by solving a convex feasibility problem, which is a smallest possible estimate set.

The remainder of this paper is organized as follows: The nonlinear SMF problem is formulated in Section II for discretetime nonlinear systems. A new nonlinear SMF algorithm for computing the state estimation ellipsoid is developed in Section III. Section IV provides an illustrative example to demonstrate the effectiveness of our algorithm. Finally, Section V draws conclusions and future directions.

Notation: The notation $X \geq Y$ (respectively, $X>Y$ ), where $X$ and $Y$ are symmetric matrices, means that $X-Y$ is positive semidefinite (respectively, positive definite). The superscript $T$ stands for matrix transposition. The notation trace $(P)$ denotes the trace of $P$.

\section{PRoblem Formulation}

Consider a class of nonlinear discrete-time systems

$$
\left\{\begin{array}{l}
x_{k+1}=f\left(x_{k}\right)+F u_{k}+g\left(x_{k}\right) w_{k} \\
y_{k}=h\left(x_{k}\right)+l\left(x_{k}\right) v_{k}
\end{array}\right.
$$

where $x_{k} \in \mathbb{R}^{n}$ is the system state; $u_{k} \in \mathbb{R}^{l}$ is the known deterministic input; $y_{k} \in \mathbb{R}^{m}$ is the measurement output; $f\left(x_{k}\right)$, $g\left(x_{k}\right), h\left(x_{k}\right)$, and $l\left(x_{k}\right)$ are the functions of $x_{k}$ with $f(0)=0$, $g(0)=0, h(0)=0$, and $l(0)=0 ; F$ is a known matrix; $w_{k} \in$ $\mathbb{R}^{r}$ is the process noise; and $v_{k} \in \mathbb{R}^{p}$ is the measurement noise, which is assumed to be confined to specified ellipsoidal sets

$$
\begin{aligned}
\mathcal{W}_{k} & =\left\{w_{k}: w_{k}^{T} Q_{k}^{-1} w_{k} \leq 1\right\} \\
\mathcal{V}_{k} & =\left\{v_{k}: v_{k}^{T} R_{k}^{-1} v_{k} \leq 1\right\}
\end{aligned}
$$

where $Q_{k}=Q_{k}^{T}>0$ and $R_{k}=R_{k}^{T}>0$ are known matrices with compatible dimensions; and the initial state $x_{0}$ belongs to a given ellipsoid

$$
\left(x_{0}-\hat{x}_{0}\right)^{T} P_{0}^{-1}\left(x_{0}-\hat{x}_{0}\right) \leq 1
$$

where $\hat{x}_{0}$ is an estimate of $x_{0}$, which is assumed to be given, and $P_{0}=P_{0}^{T}>0$ is a known matrix.

To design an appropriate filter for the nonlinear discretetime system [see (1)], the following T-S fuzzy model [30] is proposed.

Model Rule $i$ : IF $\theta_{1}(k)$ is $\mu_{i 1}$ and $\theta_{2}(k)$ is $\mu_{i 2} \ldots$ and $\theta_{p}(k)$ is $\mu_{i p}$, THEN

$$
\left\{\begin{array}{l}
x_{k+1}=A_{i} x_{k}+F u_{k}+B_{i} w_{k} \\
y_{k}=C_{i} x_{k}+D_{i} v_{k}, \quad i=1,2, \ldots, r
\end{array}\right.
$$

where $\mu_{i j}(j=1,2, \ldots, p)$ is the fuzzy set; $r$ is the number of IF-THEN rules; and $A_{i}, B_{i}, C_{i}$, and $D_{i}$ are known constant matrices with appropriate dimensions. $\left\{\theta_{1}(k), \theta_{2}(k), \ldots, \theta_{p}(k)\right\}$ are premise variables that may be a function of the state variables. Let $\theta_{k}=\left[\theta_{1}(k), \theta_{2}(k), \ldots, \theta_{p}(k)\right]$. By using the fuzzy inference methods with singleton fuzzier and weighted average defuzzier, the overall fuzzy model for the system can be inferred as follows:

$$
\left\{\begin{array}{l}
x_{k+1}=\sum_{i=1}^{r} h_{i}\left(\theta_{k}\right) A_{i} x_{k}+\sum_{i=1}^{r} h_{i}\left(\theta_{k}\right) B_{i} w_{k}+F u_{k} \\
y_{k}=\sum_{i=1}^{r} h_{i}\left(\theta_{k}\right) C_{i} x_{k}+\sum_{i=1}^{r} h_{i}\left(\theta_{k}\right) D_{i} v_{k}
\end{array}\right.
$$

where $h_{i}\left(\theta_{k}\right)=\left(\psi_{i}(\mu(k)) / \sum_{i=1}^{r} \psi_{i}(\mu(k))\right) \geq 0$ is the normalized weight for each rule with $\psi_{i}(\mu(k))=\prod_{j=1}^{p} \mu_{i j}\left(\theta_{j}(k)\right) \geq$ 0 and $\sum_{i=1}^{r} h_{i}\left(\theta_{k}\right)=1$.

The fuzzy model [see (6)] can be interpreted as an interpolation of $r$ linear systems through the membership function $h_{i}\left(\theta_{k}\right)$ to approximate the nonlinear system [see (1)]. Therefore, the nonlinear system [see (1)] can be described as

$$
\left\{\begin{aligned}
x_{k+1}= & f\left(x_{k}\right)+F u_{k}+g\left(x_{k}\right) w_{k} \\
= & \sum_{i=1}^{r} h_{i}\left(\theta_{k}\right) A_{i} x_{k}+\Delta f\left(x_{k}\right)+F u_{k} \\
& \quad+\sum_{i=1}^{r} h_{i}\left(\theta_{k}\right) B_{i} w_{k}+\Delta g\left(x_{k}\right) w_{k} \\
y_{k}= & h\left(x_{k}\right)+l\left(x_{k}\right) v_{k} \\
= & \sum_{i=1}^{r} h_{i}\left(\theta_{k}\right) C_{i} x_{k}+\Delta h\left(x_{k}\right) \\
& +\sum_{i=1}^{r} h_{i}\left(\theta_{k}\right) D_{i} v_{k}+\Delta l\left(x_{k}\right) v_{k}
\end{aligned}\right.
$$

where

$$
\left\{\begin{array}{l}
\Delta f\left(x_{k}\right)=f\left(x_{k}\right)-\sum_{i=1}^{r} h_{i}\left(\theta_{k}\right) A_{i} x_{k} \\
\Delta g\left(x_{k}\right)=g\left(x_{k}\right)-\sum_{i=1}^{r} h_{i}\left(\theta_{k}\right) B_{i} \\
\Delta h\left(x_{k}\right)=h\left(x_{k}\right)-\sum_{i=1}^{r} h_{i}\left(\theta_{k}\right) C_{i} x_{k} \\
\Delta l\left(x_{k}\right)=l\left(x_{k}\right)-\sum_{i=1}^{r} h_{i}\left(\theta_{k}\right) D_{i}
\end{array}\right.
$$

denote the approximation (or interpolation) errors between the nonlinear system [see (1)] and the fuzzy model [see (6)]. 
To take full advantage of using the fuzzy model [see (6)] for the nonlinear system [see (1)], we make the following assumptions:

$$
\left\{\begin{array}{l}
\Delta f\left(x_{k}\right)=H_{1} \Delta_{1} E_{1} x_{k} \\
\Delta g\left(x_{k}\right)=H_{2} \Delta_{2} E_{2} \\
\Delta h\left(x_{k}\right)=H_{3} \Delta_{3} E_{3} x_{k} \\
\Delta l\left(x_{k}\right)=H_{4} \Delta_{4} E_{4}
\end{array}\right.
$$

where $H_{1}, H_{2}, H_{3}, H_{4}, E_{1}, E_{2}, E_{3}$, and $E_{4}$ are known matrices; and $\Delta_{1}, \Delta_{2}, \Delta_{3}$, and $\Delta_{4}$ are unknown but bounded with $\left\|\Delta_{1}\right\| \leq 1, \quad\left\|\Delta_{2}\right\| \leq 1, \quad\left\|\Delta_{3}\right\| \leq 1, \quad$ and $\quad\left\|\Delta_{4}\right\| \leq 1$, respectively.

The T-S fuzzy models have been proved to be approximators for nonlinear systems [30], [37], [43]. However, there exists an approximation error between the fuzzy model and the original nonlinear system. For an affine nonlinear system, an approximation scheme in [42] was proposed to construct its T-S fuzzy approximator, and the corresponding approximation error bound has been determined. Therefore, we use normbounded uncertainties [see (9)] to represent the approximation error [6]. The upper bound of the norm-bounded uncertainty can be thought of as the worst-case approximation error [34]. Now, a robust fuzzy filter for the nonlinear system to tolerate the approximation error based on the assumptions [see (9)] can be designed as follows.

Filter Rule $i$ : IF $\hat{\theta}_{1}(k)$ is $\mu_{i 1}$ and $\hat{\theta}_{2}(k)$ is $\mu_{i 2}, \ldots$ and $\hat{\theta}_{p}(k)$ is $\mu_{i p}$, THEN

$$
\hat{x}_{k+1}=G_{i} \hat{x}_{k}+F u_{k}+L_{i} y_{k}, \quad i=1,2, \ldots, r
$$

where $G_{i}$ and $L_{i}$ are the fuzzy filter parameters to be determined, and $\hat{\theta}_{k}=\left\{\hat{\theta}_{1}(k), \hat{\theta}_{2}(k), \ldots, \hat{\theta}_{p}(k)\right\}$ are premise variables, which maybe functions of the state estimates. The overall fuzzy filter can be written from (10) as [11], [33]

$$
\hat{x}_{k+1}=\sum_{i=1}^{r} h_{i}\left(\hat{\theta}_{k}\right) G_{i} \hat{x}_{k}+\sum_{i=1}^{r} h_{i}\left(\hat{\theta}_{k}\right) L_{i} y_{k}+F u_{k}
$$

Substituting (7) into (11) yields

$$
\begin{aligned}
\hat{x}_{k+1}= & \sum_{i=1}^{r} h_{i}\left(\hat{\theta}_{k}\right) G_{i} \hat{x}_{k}+\sum_{i=1}^{r} h_{i}\left(\hat{\theta}_{k}\right) \\
& \times L_{i}\left[\sum_{j=1}^{r} h_{j}\left(\theta_{k}\right) C_{j} x_{k}+\Delta h\left(x_{k}\right)\right. \\
& \left.+\sum_{j=1}^{r} h_{j}\left(\theta_{k}\right) D_{j} v_{k}+\Delta l\left(x_{k}\right) v_{k}\right]+F u_{k} \\
= & \sum_{i=1}^{r} h_{i}\left(\hat{\theta}_{k}\right) G_{i} \hat{x}_{k}+\sum_{i=1}^{r} h_{i}\left(\hat{\theta}_{k}\right) \sum_{j=1}^{r} h_{j}\left(\theta_{k}\right) \\
& \times\left[L_{i}\left(C_{j} x_{k}+D_{j} v_{k}\right)\right]+\sum_{i=1}^{r} h_{i}\left(\hat{\theta}_{k}\right) \\
& \times L_{i}\left[\Delta h\left(x_{k}\right)+\Delta l\left(x_{k}\right) v_{k}\right]+F u_{k} .
\end{aligned}
$$

On the other hand, if $\left(x_{k}-\hat{x}_{k}\right)^{T} P_{k}^{-1}\left(x_{k}-\hat{x}_{k}\right) \leq 1$, then there exists a $z$ with $\|z\| \leq 1$ such that

$$
x_{k}=\hat{x}_{k}+\Xi_{k} z
$$

where $\Xi_{k}$ is a factorization of $P_{k}=\Xi_{k} \Xi_{k}^{T}$.

Thus, the state estimation error is derived from (7), (9), (12), and (13) as follows:

$$
\begin{aligned}
x_{k+1} & -\hat{x}_{k+1} \\
= & \sum_{j=1}^{r} h_{j}\left(\theta_{k}\right) A_{j} x_{k}+\Delta f\left(x_{k}\right)+\sum_{j=1}^{r} h_{j}\left(\theta_{k}\right) B_{j} w_{k} \\
& +\Delta g\left(x_{k}\right) w_{k}-\sum_{i=1}^{r} h_{i}\left(\hat{\theta}_{k}\right) G_{i} \hat{x}_{k} \\
& -\sum_{i=1}^{r} h_{i}\left(\hat{\theta}_{k}\right) \sum_{j=1}^{r} h_{j}\left(\theta_{k}\right)\left[L_{j}\left(C_{i} x_{k}+D_{i} v_{k}\right)\right] \\
& -\sum_{i=1}^{r} h_{i}\left(\hat{\theta}_{k}\right) L_{i}\left[\Delta h\left(x_{k}\right)+\Delta l\left(x_{k}\right) v_{k}\right] \\
= & \sum_{j=1}^{r} h_{j}\left(\theta_{k}\right) A_{j} x_{k}-\sum_{i=1}^{r} h_{i}\left(\hat{\theta}_{k}\right) G_{i} \hat{x}_{k}-\sum_{i=1}^{r} h_{i}\left(\hat{\theta}_{k}\right) \\
& \times \sum_{j=1}^{r} h_{j}\left(\theta_{k}\right) L_{i} C_{j} x_{k}+\sum_{j=1}^{r} h_{j}\left(\theta_{k}\right) B_{j} w_{k}-\sum_{i=1}^{r} h_{i}\left(\hat{\theta}_{k}\right) \\
& \times \sum_{j=1}^{r} h_{j}\left(\theta_{k}\right) L_{i} D_{j} v_{k}+H_{1} \Delta_{1} E_{1} x_{k}+H_{2} \Delta_{2} E_{2} w_{k} \\
& \quad-\sum_{i=1}^{r} h_{i}\left(\hat{\theta}_{k}\right) L_{i}\left(H_{3} \Delta_{3} E_{3} x_{k}+H_{4} \Delta_{4} E_{4} v_{k}\right) \\
= & \sum_{j=1}^{r} h_{j}\left(\theta_{k}\right) A_{j} \hat{x}_{k}+\sum_{j=1}^{r} h_{j}\left(\theta_{k}\right) A_{j} \Xi_{k} z \\
& -\sum_{i=1}^{r} h_{i}\left(\hat{\theta}_{k}\right) G_{i} \hat{x}_{k}-\sum_{i=1}^{r} h_{i}\left(\hat{\theta}_{k}\right) \sum_{j=1}^{r} h_{j}\left(\theta_{k}\right) L_{i} C_{j} \hat{x}_{k} \\
& -\sum_{i=1}^{r} h_{i}\left(\hat{\theta}_{k}\right) \sum_{j=1}^{r} h_{j}\left(\theta_{k}\right) L_{i} C_{j} \Xi_{k} z+\sum_{j} \Xi_{k} z+H_{j}\left(\theta_{k}\right) B_{j} w_{k} \\
& \left.+H_{i}\left(\hat{\theta}_{k}\right) \sum_{j=1}^{r} h_{j}\left(\theta_{k}\right) L_{i} D_{j} v_{k}\right) .(14) v_{k}+H_{1} \Delta_{1} E_{1} \hat{x}_{k} \\
& \\
& \\
& \\
& \\
&
\end{aligned}
$$

Defining new variables as

$$
\left\{\begin{array}{l}
q_{1}=\Delta_{1} E_{1} \hat{x}_{k} \\
q_{2}=\Delta_{1} E_{1} \Xi_{k} z \\
q_{3}=\Delta_{2} E_{2} w_{k} \\
q_{4}=\Delta_{3} E_{3} \hat{x}_{k} \\
q_{5}=\Delta_{3} E_{3} \Xi_{k} z \\
q_{6}=\Delta_{4} E_{4} v_{k}
\end{array}\right.
$$


we obtain

$$
\begin{aligned}
x_{k+1} & -\hat{x}_{k+1} \\
= & \sum_{j=1}^{r} h_{j}\left(\theta_{k}\right) A_{j} \hat{x}_{k}+\sum_{j=1}^{r} h_{j}\left(\theta_{k}\right) A_{j} \Xi_{k} z-\sum_{i=1}^{r} h_{i}\left(\hat{\theta}_{k}\right) G_{i} \hat{x}_{k} \\
& -\sum_{i=1}^{r} h_{i}\left(\hat{\theta}_{k}\right) \sum_{j=1}^{r} h_{j}\left(\theta_{k}\right) L_{i} C_{j} \hat{x}_{k}-\sum_{i=1}^{r} h_{i}\left(\hat{\theta}_{k}\right) \\
& \times \sum_{j=1}^{r} h_{j}\left(\theta_{k}\right) L_{i} C_{j} \Xi_{k} z+\sum_{j=1}^{r} h_{j}\left(\theta_{k}\right) B_{j} w_{k}-\sum_{i=1}^{r} h_{i}\left(\hat{\theta}_{k}\right) \\
& \times \sum_{j=1}^{r} h_{j}\left(\theta_{k}\right) L_{i} D_{j} v_{k}+H_{1} q_{1}+H_{1} q_{2}+H_{2} q_{3} \\
& -\sum_{i=1}^{r} h_{i}\left(\hat{\theta}_{k}\right) L_{i}\left(H_{3} q_{4}+H_{3} q_{5}+H_{4} q_{6}\right) .
\end{aligned}
$$

Denoting

$$
\eta=\left[\begin{array}{c}
1 \\
z \\
w_{k} \\
v_{k} \\
q_{1} \\
q_{2} \\
q_{3} \\
q_{4} \\
q_{5} \\
q_{6}
\end{array}\right]
$$

and noting the fact that $\sum_{i=1}^{r} h_{i}\left(\hat{\theta}_{k}\right)=1$, we have

$$
x_{k+1}-\hat{x}_{k+1}=\sum_{i=1}^{r} h_{i}\left(\hat{\theta}_{k}\right) \sum_{j=1}^{r} h_{j}\left(\theta_{k}\right) \Phi_{i j} \eta
$$

where

$$
\begin{gathered}
\Phi_{i j}=\left[\begin{array}{ccccc}
A_{j} \hat{x}_{k}-G_{i} \hat{x}_{k}-L_{i} C_{j} \hat{x}_{k} & A_{j} \Xi_{k}-L_{i} C_{j} \Xi_{k} \\
B_{j}-L_{i} D_{j} & H_{1} & H_{1} & H_{2} & -L_{i} H_{3} \\
-L_{i} H_{3} & \left.-L_{i} H_{4}\right] .
\end{array}\right.
\end{gathered}
$$

Then

$$
\begin{aligned}
\left(x_{k+1}-\hat{x}_{k+1}\right)^{T} P_{k+1}^{-1}\left(x_{k+1}-\hat{x}_{k+1}\right) & \\
= & \eta^{T} \sum_{i=1}^{r} h_{i}\left(\hat{\theta}_{k}\right) \sum_{j=1}^{r} h_{j}\left(\theta_{k}\right) \Phi_{i j}^{T} P_{k+1}^{-1} \sum_{l=1}^{r} h_{l}\left(\hat{\theta}_{k}\right) \\
& \times \sum_{m=1}^{r} h_{m}\left(\theta_{k}\right) \Phi_{l m} \eta \\
= & \sum_{i=1}^{r} h_{i}\left(\hat{\theta}_{k}\right) \sum_{j=1}^{r} h_{j}\left(\theta_{k}\right) \sum_{l=1}^{r} h_{l}\left(\hat{\theta}_{k}\right) \\
& \times \sum_{m=1}^{r} h_{m}\left(\theta_{k}\right) \eta^{T} \Phi_{i j}^{T} P_{k+1}^{-1} \Phi_{l m} \eta
\end{aligned}
$$

where $P_{k+1}$ is a design parameter, which is used to find the ellipsoidal set of possible system states $x_{k}$ such that $\left(x_{k+1}-\right.$ $\left.\hat{x}_{k+1}\right)^{T} P_{k+1}^{-1}\left(x_{k+1}-\hat{x}_{k+1}\right) \leq 1$.

Our objective in this paper is to determine a minimal ellipsoid for the state $x_{k+1}$ in some sense, given the measurement information $y_{k}$ at time instant $k$ for the process noise $w_{k} \in \mathcal{W}_{k}$, the measurement noise $v_{k} \in \mathcal{V}_{k}$, and all the uncertainties $\left\|\Delta_{1}\right\| \leq$ $1,\left\|\Delta_{2}\right\| \leq 1,\left\|\Delta_{3}\right\| \leq 1$, and $\left\|\Delta_{4}\right\| \leq 1$. In other words, we look for $P_{k+1}$ and $\hat{x}_{k+1}$ such that

$$
\min _{P_{k+1}>0} \operatorname{trace}\left(P_{k+1}\right)
$$

subject to $\quad\left(x_{k+1}-\hat{x}_{k+1}\right)^{T} P_{k+1}^{-1}\left(x_{k+1}-\hat{x}_{k+1}\right) \leq 1$

for any $w_{k} \in \mathcal{W}_{k}, v_{k} \in \mathcal{V}_{k},\left\|\Delta_{1}\right\| \leq 1,\left\|\Delta_{2}\right\| \leq 1,\left\|\Delta_{3}\right\| \leq 1$, and $\left\|\Delta_{4}\right\| \leq 1$

The above filtering problem is referred to as the nonlinear SMF problem.

\section{Solution to the Nonlinear SMF Problem}

To obtain the solution to the nonlinear SMF problem [see (21) and (22)], we get the following result from (20):

$$
\begin{aligned}
&\left(x_{k+1}-\hat{x}_{k+1}\right)^{T} P_{k+1}^{-1}\left(x_{k+1}-\hat{x}_{k+1}\right) \\
&=\sum_{i=1}^{r} h_{i}\left(\hat{\theta}_{k}\right) \sum_{j=1}^{r} h_{j}\left(\theta_{k}\right) \sum_{l=1}^{r} h_{l}\left(\hat{\theta}_{k}\right) \\
& \quad \times \sum_{m=1}^{r} h_{m}\left(\theta_{k}\right) \eta^{T} \Phi_{i j}^{T} P_{k+1}^{-1} \Phi_{l m} \eta \\
&=\sum_{i=1}^{r} h_{i}\left(\hat{\theta}_{k}\right) \sum_{l=1}^{r} h_{l}\left(\hat{\theta}_{k}\right) \\
& \quad \times\left[\sum_{j=1}^{r} h_{j}\left(\theta_{k}\right) \sum_{m=1}^{r} h_{m}\left(\theta_{k}\right) \eta^{T} \Phi_{i j}^{T} P_{k+1}^{-1} \Phi_{l m} \eta\right] \\
& \leq \sum_{i=1}^{r} h_{i}\left(\hat{\theta}_{k}\right) \sum_{l=1}^{r} h_{l}\left(\hat{\theta}_{k}\right) \sum_{j=1}^{r} h_{j}\left(\theta_{k}\right) \eta^{T} \Phi_{i j}^{T} P_{k+1}^{-1} \Phi_{l j} \eta \\
&= \sum_{j=1}^{r} h_{j}\left(\theta_{k}\right)\left[\sum_{i=1}^{r} h_{i}\left(\hat{\theta}_{k}\right) \sum_{l=1}^{r} h_{l}\left(\hat{\theta}_{k}\right) \eta^{T} \Phi_{i j}^{T} P_{k+1}^{-1} \Phi_{l j} \eta\right] \\
& \leq \sum_{j=1}^{r} h_{j}\left(\theta_{k}\right) \sum_{i=1}^{r} h_{i}\left(\hat{\theta}_{k}\right) \eta^{T} \Phi_{i j}^{T} P_{k+1}^{-1} \Phi_{i j} \eta \\
&= \sum_{i=1}^{r} h_{i}\left(\hat{\theta}_{k}\right) \sum_{j=1}^{r} h_{j}\left(\theta_{k}\right) \eta^{T} \Phi_{i j}^{T} P_{k+1}^{-1} \Phi_{i j} \eta .
\end{aligned}
$$

With $\left\|\Delta_{1}\right\| \leq 1,\left\|\Delta_{2}\right\| \leq 1,\left\|\Delta_{3}\right\| \leq 1$, and $\left\|\Delta_{4}\right\| \leq 1$, we can infer from (15) that

$$
\left\{\begin{array}{l}
q_{1}^{T} q_{1}-\hat{x}_{k}^{T} E_{1}^{T} E_{1} \hat{x}_{k} \leq 0 \\
q_{2}^{T} q_{2}-z^{T} \Xi_{k}^{T} E_{1}^{T} E_{1} \Xi_{k} z \leq 0 \\
q_{3}^{T} q_{3}-w_{k}^{T} E_{2}^{T} E_{2} w_{k} \leq 0 \\
q_{4}^{T} q_{4}-\hat{x}_{k}^{T} E_{3}^{T} E_{3} \hat{x}_{k} \leq 0 \\
q_{5}^{T} q_{5}-z^{T} \Xi_{k}^{T} E_{3}^{T} E_{3} \Xi_{k} z \leq 0 \\
q_{6}^{T} q_{6}-v_{k}^{T} E_{4}^{T} E_{4} v_{k} \leq 0 .
\end{array}\right.
$$


Thus, the unknown variables $z, w_{k}, v_{k}, q_{1}, q_{2}, q_{3}, q_{4}, q_{5}$, and $q_{6}$ satisfy the following conditions:

$$
\left\{\begin{array}{l}
\|z\| \leq 1 \\
w_{k}^{T} Q_{k}^{-1} w_{k} \leq 1 \\
v_{k}^{T} R_{k}^{-1} v_{k} \leq 1 \\
q_{1}^{T} q_{1}-\hat{x}_{k}^{T} E_{1}^{T} E_{1} \hat{x}_{k} \leq 0 \\
q_{2}^{T} q_{2}-z^{T} \Xi_{k}^{T} E_{1}^{T} E_{1} \Xi_{k} z \leq 0 \\
q_{3}^{T} q_{3}-w_{k}^{T} E_{2}^{T} E_{2} w_{k} \leq 0 \\
q_{4}^{T} q_{4}-\hat{x}_{k}^{T} E_{3}^{T} E_{3} \hat{x}_{k} \leq 0 \\
q_{5}^{T} q_{5}-z^{T} \Xi_{k}^{T} E_{3}^{T} E_{3} \Xi_{k} z \leq 0 \\
q_{6}^{T} q_{6}-v_{k}^{T} E_{4}^{T} E_{4} v_{k} \leq 0 .
\end{array}\right.
$$

We write (25) in $\eta$ as

$$
\left\{\begin{array}{l}
\eta^{T} \operatorname{diag}(-1, I, 0,0,0,0,0,0,0,0) \eta \leq 0 \\
\eta^{T} \operatorname{diag}\left(-1,0, Q_{k}^{-1}, 0,0,0,0,0,0,0\right) \eta \leq 0 \\
\eta^{T} \operatorname{diag}\left(-1,0,0, R_{k}^{-1}, 0,0,0,0,0,0\right) \eta \leq 0 \\
\eta^{T} \operatorname{diag}\left(-\hat{x}_{k}^{T} E_{1}^{T} E_{1} \hat{x}_{k}, 0,0,0, I, 0,0,0,0,0\right) \eta \leq 0 \\
\eta^{T} \operatorname{diag}\left(0,-\Xi_{k}^{T} E_{1}^{T} E_{1} \Xi_{k}, 0,0,0, I, 0,0,0,0\right) \eta \leq 0 \\
\eta^{T} \operatorname{diag}\left(0,0,-E_{2}^{T} E_{2}, 0,0,0, I, 0,0,0\right) \eta \leq 0 \\
\eta^{T} \operatorname{diag}\left(-\hat{x}_{k}^{T} E_{3}^{T} E_{3} \hat{x}_{k}, 0,0,0,0,0,0, I, 0,0\right) \eta \leq 0 \\
\eta^{T} \operatorname{diag}\left(0,-\Xi_{k}^{T} E_{3}^{T} E_{3} \Xi_{k}, 0,0,0,0,0,0, I, 0\right) \eta \leq 0 \\
\eta^{T} \operatorname{diag}\left(0,0,0,-E_{4}^{T} E_{4}, 0,0,0,0,0, I\right) \eta \leq 0 .
\end{array}\right.
$$

On the other hand, our goal from (23) and (22) is to achieve that

$$
\sum_{i=1}^{r} h_{i}\left(\hat{\theta}_{k}\right) \sum_{j=1}^{r} h_{j}\left(\theta_{k}\right) \eta^{T} \Phi_{i j}^{T} P_{k+1}^{-1} \Phi_{i j} \eta \leq 1 .
$$

Hence, the proposed problem is transferred to solve the problem in (27) subject to the inequality constraints in (26).

Now, we apply S-procedure [5] to (26) and (27). The sufficient condition such that the inequalities (26) imply (27) to hold is that there exist positive scalars $\tau_{1}, \tau_{2}, \tau_{3}, \tau_{4}, \tau_{5}, \tau_{6}, \tau_{7}, \tau_{8}$, and $\tau_{9}$ such that

$$
\begin{aligned}
\Phi_{i j}^{T} & \left.P_{k+1}^{-1} \Phi_{i j}-\operatorname{diag}(1,0,0,0,0,0,0,0,0,0)\right) \\
& -\tau_{1} \operatorname{diag}(-1, I, 0,0,0,0,0,0,0,0) \\
& -\tau_{2} \operatorname{diag}\left(-1,0, Q_{k}^{-1}, 0,0,0,0,0,0,0\right) \\
& -\tau_{3} \operatorname{diag}\left(-1,0,0, R_{k}^{-1}, 0,0,0,0,0,0\right) \\
& -\tau_{4} \operatorname{diag}\left(-\hat{x}_{k}^{T} E_{1}^{T} E_{1} \hat{x}_{k}, 0,0,0, I, 0,0,0,0,0\right) \\
& -\tau_{5} \operatorname{diag}\left(0,-\Xi_{k}^{T} E_{1}^{T} E_{1} \Xi_{k}, 0,0,0, I, 0,0,0,0\right) \\
& -\tau_{6} \operatorname{diag}\left(0,0,-E_{2}^{T} E_{2}, 0,0,0, I, 0,0,0\right) \\
& -\tau_{7} \operatorname{diag}\left(-\hat{x}_{k}^{T} E_{3}^{T} E_{3} \hat{x}_{k}, 0,0,0,0,0,0, I, 0,0\right) \\
& -\tau_{8} \operatorname{diag}\left(0,-\Xi_{k}^{T} E_{3}^{T} E_{3} \Xi_{k}, 0,0,0,0,0,0, I, 0\right) \\
& -\tau_{9} \operatorname{diag}\left(0,0,0,-E_{4}^{T} E_{4}, 0,0,0,0,0, I\right) \leq 0 .
\end{aligned}
$$

Equation (28) is written in the following compact form:

$$
\begin{aligned}
\Phi_{i j}^{T} P_{k+1}^{-1} \Phi_{i j} & \\
-\operatorname{diag}( & 1-\tau_{1}-\tau_{2}-\tau_{3}-\tau_{4}-\tau_{4} \hat{x}_{k}^{T} E_{1}^{T} E_{1} \hat{x}_{k} \\
& -\tau_{7} \hat{x}_{k}^{T} E_{3}^{T} E_{3} \hat{x}_{k}, \tau_{1} I-\tau_{5} \Xi_{k}^{T} E_{1}^{T} E_{1} \Xi_{k} \\
& -\tau_{8} \Xi_{k}^{T} E_{3}^{T} E_{3} \Xi_{k}, \tau_{2} Q_{k}^{-1}-\tau_{6} E_{2}^{T} E_{2}, \tau_{3} R_{k}^{-1} \\
& \left.-\tau_{9} E_{4}^{T} E_{4}, \tau_{4} I, \tau_{5} I, \tau_{6} I, \tau_{7} I, \tau_{8} I, \tau_{9} I\right) \leq 0 .
\end{aligned}
$$

By denoting

$$
\begin{gathered}
\Theta\left(\tau_{1}, \tau_{2}, \tau_{3}, \tau_{4}, \tau_{5}, \tau_{6}, \tau_{7}, \tau_{8}, \tau_{9}\right) \\
=\operatorname{diag}\left(1-\tau_{1}-\tau_{2}-\tau_{3}-\tau_{4}-\tau_{4} \hat{x}_{k}^{T} E_{1}^{T} E_{1} \hat{x}_{k}\right. \\
\quad-\tau_{7} \hat{x}_{k}^{T} E_{3}^{T} E_{3} \hat{x}_{k}, \tau_{1} I-\tau_{5} \Xi_{k}^{T} E_{1}^{T} E_{1} \Xi_{k} \\
\quad-\tau_{8} \Xi_{k}^{T} E_{3}^{T} E_{3} \Xi_{k}, \tau_{2} Q_{k}^{-1}-\tau_{6} E_{2}^{T} E_{2}, \tau_{3} R_{k}^{-1} \\
\left.\quad-\tau_{9} E_{4}^{T} E_{4}, \tau_{4} I, \tau_{5} I, \tau_{6} I, \tau_{7} I, \tau_{8} I, \tau_{9} I\right)
\end{gathered}
$$

we can write (30) as

$$
\Phi_{i j}^{T} P_{k+1}^{-1} \Phi_{i j}-\Theta\left(\tau_{1}, \tau_{2}, \tau_{3}, \tau_{4}, \tau_{5}, \tau_{6}, \tau_{7}, \tau_{8}, \tau_{9}\right) \leq 0
$$

By using Schur complements [29], (31) is equivalent to

$$
\left[\begin{array}{cc}
-P_{k+1} & \Phi_{i j} \\
\Phi_{i j}^{T} & -\Theta\left(\tau_{1}, \tau_{2}, \tau_{3}, \tau_{4}, \tau_{5}, \tau_{6}, \tau_{7}, \tau_{8}, \tau_{9}\right)
\end{array}\right] \leq 0
$$

Hence, we can get the following theorem.

Theorem 1: For the system [see (1)] that satisfies the assumptions [see (9)] and that its state $x_{k}$ belongs to the state estimation ellipsoid $\left(x_{k}-\hat{x}_{k}\right)^{T} P_{k}^{-1}\left(x_{k}-\hat{x}_{k}\right) \leq 1$, where $\hat{x}_{k}$ and $P_{k}>0$ are known, if there exist a symmetric positive definite matrix $P_{k+1}=P_{k+1}^{T}>0$, real matrices $G_{i}$ and $L_{i}$, and positive scalars $\tau_{1}>0, \tau_{2}>0, \tau_{3}>0, \tau_{4}>0, \tau_{5}>0, \tau_{6}>0$, $\tau_{7}>0, \tau_{8}>0$, and $\tau_{9}>0$ such that the following linear matrix inequality (LMI):

$$
\left[\begin{array}{cc}
-P_{k+1} & \Phi_{i j} \\
\Phi_{i j}^{T} & -\Theta\left(\tau_{1}, \tau_{2}, \tau_{3}, \tau_{4}, \tau_{5}, \tau_{6}, \tau_{7}, \tau_{8}, \tau_{9}\right)
\end{array}\right] \leq 0
$$

holds for all $i, j=1,2, \ldots, r$, then a one-step ahead state $x_{k+1}$ will reside in its state estimation ellipsoid $\left(x_{k+1}-\right.$ $\left.\hat{x}_{k+1}\right)^{T} P_{k+1}^{-1}\left(x_{k+1}-\hat{x}_{k+1}\right) \leq 1$, where

$$
\begin{aligned}
& \Phi_{i j}=\left[A_{j} \hat{x}_{k}-G_{i} \hat{x}_{k}-L_{i} C_{j} \hat{x}_{k} \quad A_{j} \Xi_{k}-L_{i} C_{j} \Xi_{k}\right. \\
& B_{j} \quad-L_{i} D_{j} \quad H_{1} \quad H_{1} \quad H_{2} \quad-L_{i} H_{3} \\
& \text { - } \left.L_{i} H_{3} \quad-L_{i} H_{4}\right]
\end{aligned}
$$

and

$$
\begin{aligned}
\Theta\left(\tau_{1}, \tau_{2}, \tau_{3}, \tau_{4}, \tau_{5}, \tau_{6}, \tau_{7}, \tau_{8}, \tau_{9}\right) & \operatorname{diag}\left(1-\tau_{1}-\tau_{2}-\tau_{3}-\tau_{4}-\tau_{4} \hat{x}_{k}^{T} E_{1}^{T} E_{1} \hat{x}_{k}\right. \\
& -\tau_{7} \hat{x}_{k}^{T} E_{3}^{T} E_{3} \hat{x}_{k}, \tau_{1} I-\tau_{5} \Xi_{k}^{T} E_{1}^{T} E_{1} \Xi_{k} \\
& -\tau_{8} \Xi_{k}^{T} E_{3}^{T} E_{3} \Xi_{k}, \tau_{2} Q_{k}^{-1}-\tau_{6} E_{2}^{T} E_{2}, \tau_{3} R_{k}^{-1} \\
& \left.-\tau_{9} E_{4}^{T} E_{4}, \tau_{4} I, \tau_{5} I, \tau_{6} I, \tau_{7} I, \tau_{8} I, \tau_{9} I\right) .
\end{aligned}
$$


Proof: If there exist $P_{k+1}>0, G_{i}, L_{i}, \tau_{1}>0, \tau_{2}>0$, $\tau_{3}>0, \tau_{4}>0, \tau_{5}>0, \tau_{6}>0, \tau_{7}>0, \tau_{8}>0$, and $\tau_{9}>0$ such that (33) holds for all $i, j=1,2, \ldots, r$, then we have

$$
\sum_{i=1}^{r} h_{i}\left(\hat{\theta}_{k}\right) \sum_{j=1}^{r} h_{j}\left(\theta_{k}\right) \eta^{T} \Phi_{i j}^{T} P_{k+1}^{-1} \Phi_{i j} \eta \leq 1 .
$$

From (23), we obtain

$$
\left(x_{k+1}-\hat{x}_{k+1}\right)^{T} P_{k+1}^{-1}\left(x_{k+1}-\hat{x}_{k+1}\right) \leq 1
$$

which completes the proof.

Theorem 1 provides a sufficient condition [see (33)] to guarantee that the state resides in its state estimation ellipsoid $\left(x_{k+1}-\hat{x}_{k+1}\right)^{T} P_{k+1}^{-1}\left(x_{k+1}-\hat{x}_{k+1}\right) \leq 1$. It also requires that the previous state stays in the previous state estimation ellipsoid $\left(x_{k}-\hat{x}_{k}\right)^{T} P_{k}^{-1}\left(x_{k}-\hat{x}_{k}\right) \leq 1$. We can, therefore, construct a recursive algorithm to implement Theorem 1. Moreover, since $P_{k+1}$ is linear in LMI [see (33)], it can be included as an optimization variable, which can be exploited to obtain a minimal state estimation ellipsoid. The optimization problem is formed as follows:

$$
\begin{aligned}
\min _{P_{k+1}>0, G_{i}, L_{i}, \tau_{1}>0, \tau_{2}>0, \tau_{3}>0, \tau_{4}>0, \tau_{5}>0, \tau_{6}>0, \tau_{7}>0, \tau_{8}>0, \tau_{9}>0} & \operatorname{trace}\left(P_{k+1}\right)
\end{aligned}
$$

subject to

$$
\begin{gathered}
{\left[\begin{array}{cc}
-P_{k+1} & \Phi_{i j} \\
\Phi_{i j}^{T} & -\Theta\left(\tau_{1}, \tau_{2}, \tau_{3}, \tau_{4}, \tau_{5}, \tau_{6}, \tau_{7}, \tau_{8}, \tau_{9}\right)
\end{array}\right] \leq 0} \\
i, j=1,2, \ldots, r .
\end{gathered}
$$

Equations (38) and (39) provide the computation of the state estimation ellipsoid of the minimal size in the sense of trace. Now, we summarize the above recursive algorithm as follows.

\section{The Nonlinear SMF Recursive Algorithm}

Step 1) Given the initial values $\left(\hat{x}_{0}, P_{0}\right), k=0$, and the finite time horizon $K$.

Step 2) Compute the shape of the state estimation ellipsoid $P_{k+1}$ and filter parameters $G_{i}$ and $L_{i}$ by solving the optimization problem (38) and (39).

Step 3) If $k=K$, then stop; otherwise, $k=k+1$, and go to Step 2).

Remark 1: We can see from Theorem 1 that the inequality [see (33)] is linear to the variables $P_{k+1}, G_{i}$ and $L_{i}, \tau_{1}, \tau_{2}, \tau_{3}$, $\tau_{4}, \tau_{5}, \tau_{6}, \tau_{7}, \tau_{8}$, and $\tau_{9}$. Hence, the optimization problem in (38) subject to (39) can be solved by the existing semidefinite programming (SDP) via interior-point approach [21], [35]. The algorithm provided is recursive and finite-horizon time. Therefore, we only guarantee the convergence of the state estimate ellipsoid for finite-horizon time. The ellipsoid set is determined by computing $P_{k+1}$ step by step according to the noises and the approximation errors.

Remark 2: Another good measure of the ellipsoid is to choose $\log \operatorname{det}\left(P_{k+1}\right)$ as the objective function. However, if we change the objective function trace $\left(P_{k+1}\right)$ to $\log \operatorname{det}\left(P_{k+1}\right)$, then the optimization problem [see (38)] is not convex. The existing SDP cannot be used to solve the nonconvex optimiza- tion problem. To transfer this nonconvex optimization problem into a convex optimization one, a decoupled technique has been proposed in [13], which provides a unique optimal ellipsoid. Other measures of the ellipsoid can also be introduced, for example, the maximum singular value of $P_{k+1}$.

\section{Simulation Example}

Consider the following discrete-time nonlinear system:

$$
\begin{aligned}
x_{1, k+1} & =0.2 x_{1, k}-0.3\left(x_{2, k}-x_{1, k}^{2}\right)+w_{k} \\
x_{1, k+1} & =0.3 x_{1, k}+0.2\left(x_{2, k}-x_{1, k}^{2}\right)+w_{k} \\
y_{k} & =x_{1, k}+0.1 x_{1, k}^{2}+x_{2, k}+0.1 x_{2, k}^{2}+v_{k}
\end{aligned}
$$

where the state $x_{k}=\left[\begin{array}{ll}x_{1, k} & x_{2, k}\end{array}\right]^{T}$.

Now, we construct the following fuzzy models to approximate the above nonlinear system:

- Rule 1: IF $x_{1, k}$ is about 1 ,THEN $x_{k+1}=A_{1} x_{k}+B_{1} w_{k}$, $y_{k}=C_{1} x_{k}+D_{1} v_{k}$

- Rule 2: IF $x_{1, k}$ is about 0,THEN $x_{k+1}=A_{2} x_{k}+B_{2} w_{k}$, $y_{k}=C_{2} x_{k}+D_{2} v_{k}$

where

$$
\begin{aligned}
& A_{1}=\left[\begin{array}{cc}
0.5 & -0.3 \\
0.1 & 0.2
\end{array}\right] \quad B_{1}=\left[\begin{array}{l}
1 \\
1
\end{array}\right] \quad C_{1}=\left[\begin{array}{ll}
1.1 & 1.1
\end{array}\right] \quad D_{1}=1 \\
& A_{2}=\left[\begin{array}{cc}
0.2 & -0.3 \\
0.3 & 0.2
\end{array}\right] \quad B_{2}=\left[\begin{array}{l}
1 \\
1
\end{array}\right] \quad C_{2}=\left[\begin{array}{ll}
1.0 & 1.0
\end{array}\right] \quad D_{2}=1 .
\end{aligned}
$$

For the convenience of simulation, triangular membership functions are used for Rules 1 and 2 in this example.

In the above fuzzy models, the approximation errors between the nonlinear system and the fuzzy models are assumed to satisfy (9), where

$$
\begin{array}{llll}
H_{1}=\left[\begin{array}{l}
0.1 \\
0.1
\end{array}\right] & E_{1}=\left[\begin{array}{ll}
0 & 0.5
\end{array}\right] & H_{2}=\left[\begin{array}{l}
0 \\
0
\end{array}\right] \quad E_{2}=0 \\
H_{3}=0.1 & E_{3}=\left[\begin{array}{lll}
0 & 0.5
\end{array}\right] & H_{4}=0 & E_{4}=0 .
\end{array}
$$

In the simulation, $w_{k}$ and $v_{k}$ are chosen as $0.5 \sin (2 k)$ and $0.5 \sin (30 k)$, respectively. The initial state is set as $x_{0}=[00]^{T}$, which belongs to the ellipsoid $\left(x_{0}-\hat{x}_{0}\right)^{T} P_{0}^{-1}\left(x_{0}-\hat{x}_{0}\right) \leq 1$, where $\hat{x}_{0}=\left[\begin{array}{ll}1 & 1\end{array}\right]^{T}$, and $P_{0}=\left[\begin{array}{cc}50 & 0 \\ 0 & 50\end{array}\right], Q_{k}=1-k / 100$, and $R_{k}=1-k / 100$.

The simulation results are obtained by solving the convex optimization problem in (38) subject to (39) under Matlab 6.5 with YALMIP 3.0 and SeDuMi 1.1 [17]. Fig. 1 shows the phase-plane estimation using the proposed SMF. We can see that the true states are always contained in the estimated ellipsoid. It is also seen in Figs. 2 and 3 that the true states reside between the upper and lower bounds.

Since we cannot exactly know how much the approximation errors between the nonlinear system and the fuzzy models, we always overbound the approximation error bounds. Now, we 


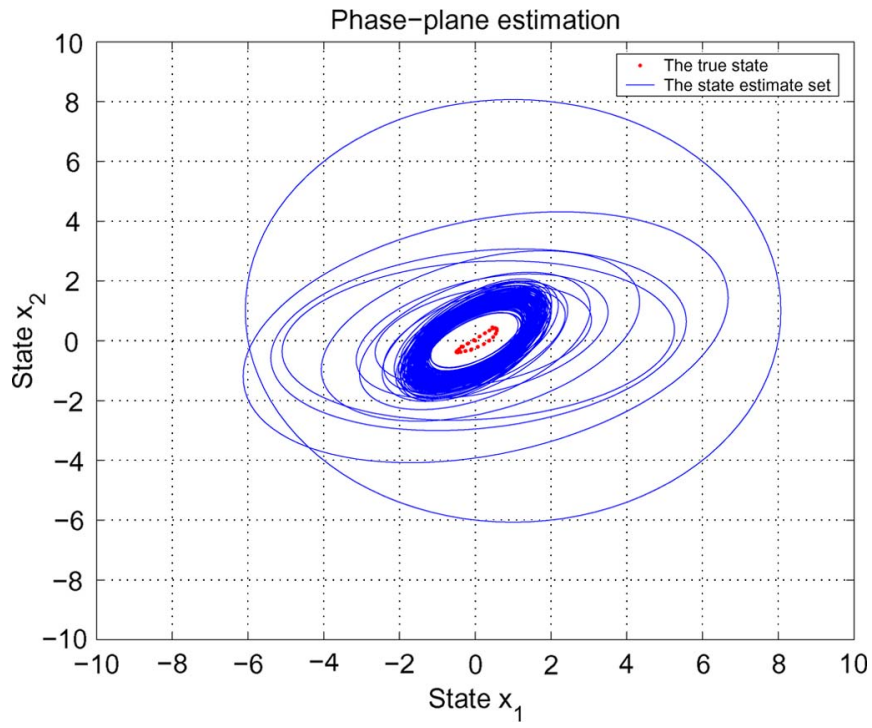

Fig. 1. Phase-plane estimation using the proposed SMF for a nonlinear system with small approximation error bounds.

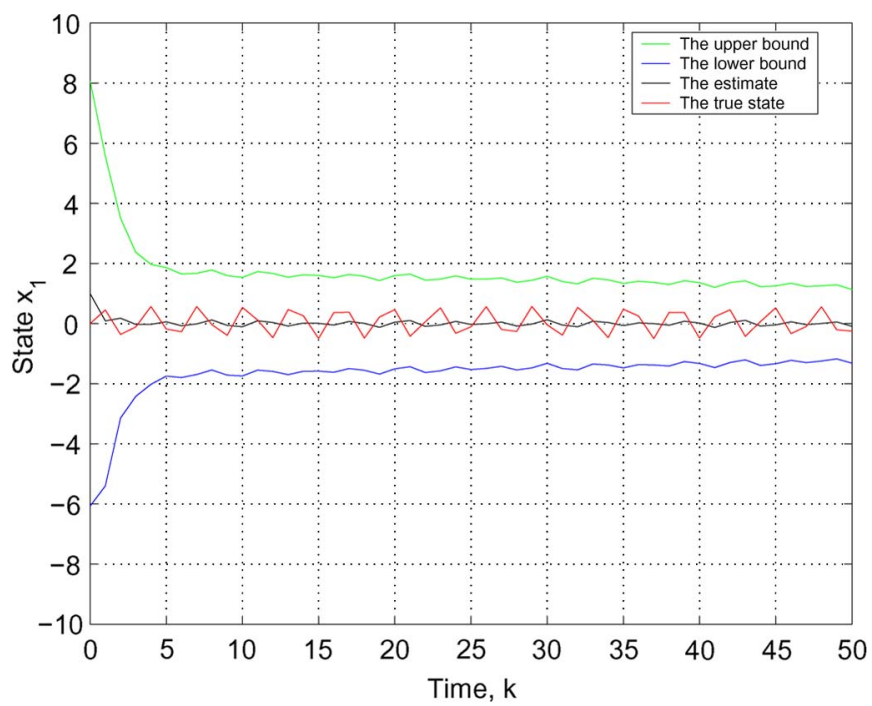

Fig. 2. True state value, state estimation, and its bounds for a nonlinear system with small approximation error bounds.

shall investigate the impacts of the overbound on the simulation results. For example, we assume the large bounds:

$$
\begin{aligned}
& H_{1}=\left[\begin{array}{l}
0.2 \\
0.2
\end{array}\right] \quad E_{1}=\left[\begin{array}{ll}
0 & 1
\end{array}\right] \quad H_{2}=\left[\begin{array}{l}
0 \\
0
\end{array}\right] \quad E_{2}=0 \\
& H_{3}=0.2 \quad E_{3}=\left[\begin{array}{ll}
0 & 1
\end{array}\right] \quad H_{4}=0 \quad E_{4}=0 .
\end{aligned}
$$

We resolve the optimization problem in (38) subject to (39) under Matlab 6.5 with YALMIP 3.0 and SeDuMi 1.1 [17]. We also obtain the phase-plane estimation shown in Fig. 4 and the true states, state estimates, and upper and lower bounds shown in Figs. 5 and 6. From the simulation results, we can see that the upper and lower bounds are bigger than those with the small approximation error bounds. Thus, the conservative bound estimate for the approximation errors will bring the bigger upper and lower bounds for the true states.

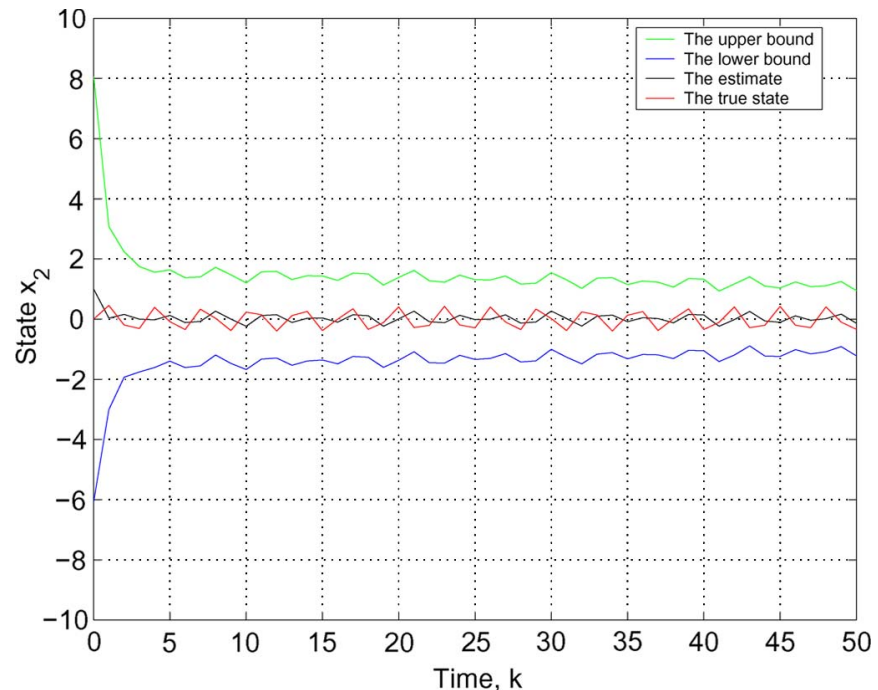

Fig. 3. True state value, state estimation, and its bounds for a nonlinear system with small approximation error bounds.

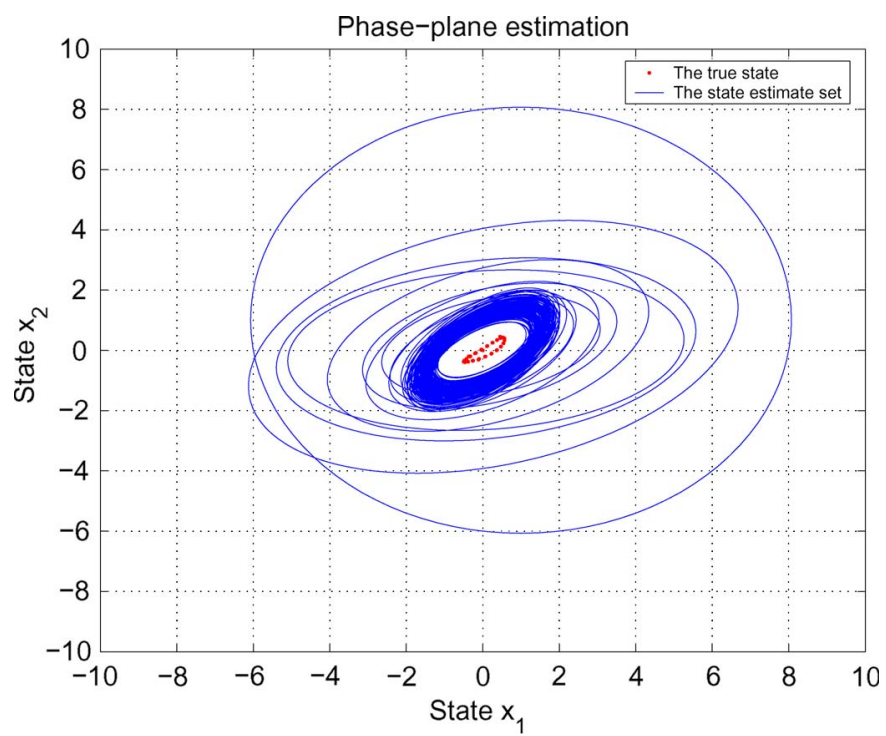

Fig. 4. Phase-plane estimation using the proposed SMF for a nonlinear system with large approximation error bounds.

\section{CONCLUSION}

This paper has provided a new SMF method for discrete-time nonlinear systems. We have employed the T-S fuzzy model to approximate the nonlinear systems over the true value of state and to overcome the difficulty with the linearization over a state estimate set rather than a state estimate point in the setmembership framework. Based on the T-S fuzzy model, we have applied the fuzzy modeling approach and the $S$-procedure to determine a state estimation ellipsoid that is a set of states compatible with the measurements, the unknown-but-bounded process and measurement noises, and the modeling approximation errors. A recursive algorithm has been derived for computing the ellipsoid that guarantees to contain the true state. An illustrative example has demonstrated the feasibility of the proposed filtering methods. The algorithm is computationally attractive for online systems with nonlinearities in the presence of unknown-but-bounded process and measurement noises. The 


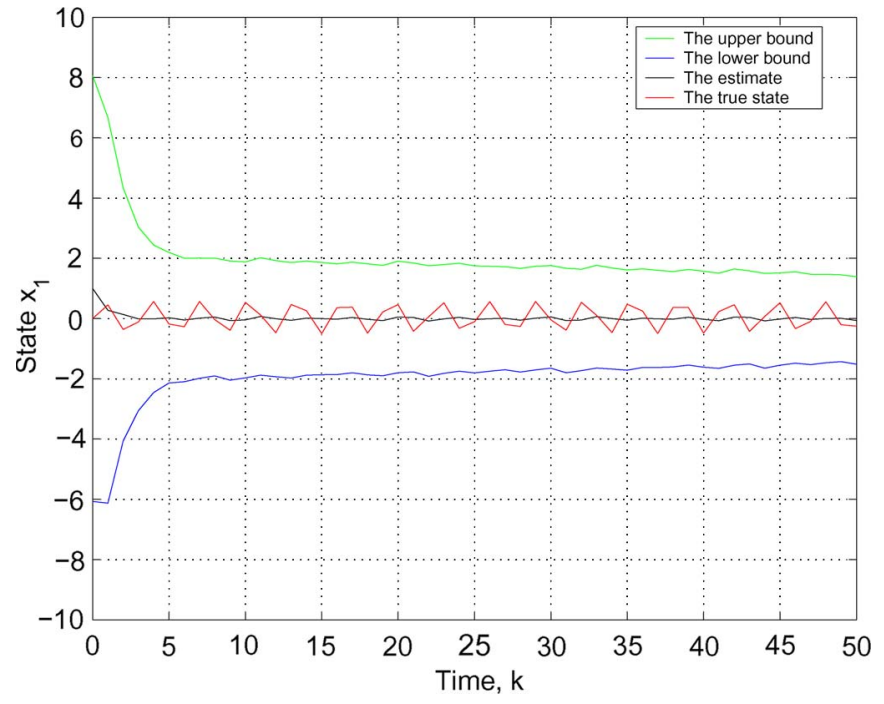

Fig. 5. True state value, state estimation, and its bounds for a nonlinear system with large approximation error bounds.

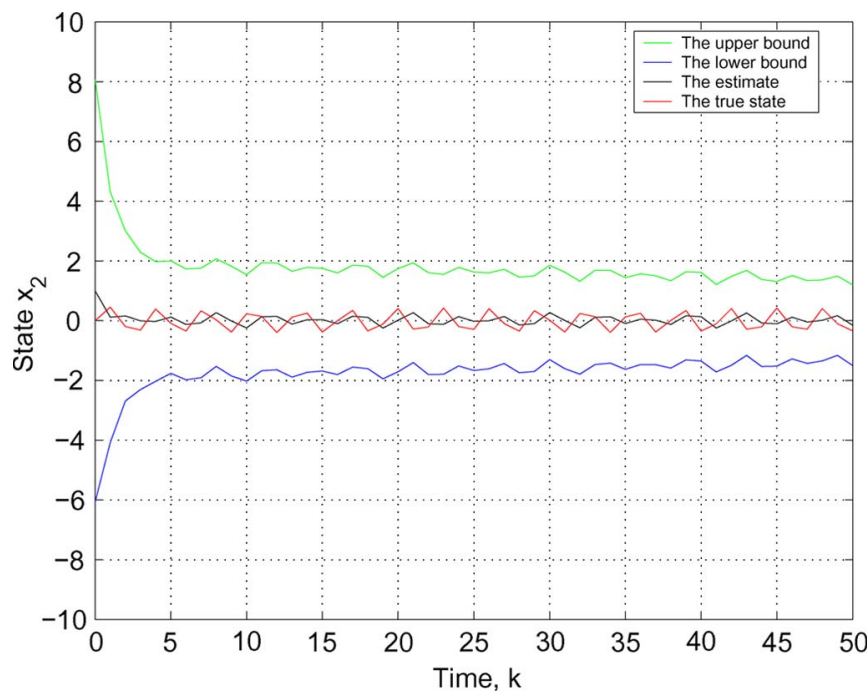

Fig. 6. True state value, state estimation, and its bounds for a nonlinear system with large approximation error bounds.

future research topics will focus on the study of the convergence of the algorithms and how to reduce the conservatism of the possible estimation sets.

\section{ACKNOWLEDGMENT}

The authors would like to thank the Associate Editor and the anonymous reviewers for their valuable comments which help improve the paper in many aspects.

\section{REFERENCES}

[1] D. Alspach and H. Sorenson, "Nonlinear Bayesian estimation using Gaussian sum approximations," IEEE Trans. Autom. Control, vol. AC-17, no. 4, pp. 439-448, Aug. 1972.

[2] B. D. O. Anderson and J. B. Moore, Optimal Filtering. Englewood Cliffs, NJ: Prentice-Hall, 1979.

[3] D. P. Bertsekas and I. B. Rhodes, "Recursive state estimation for a setmembership description of uncertainty," IEEE Trans. Autom. Control, vol. AC-16, no. 2, pp. 117-128, Apr. 1971.
[4] E. Bølviken, P. J. Acklam, N. Christophersen, and J.-M. Størdal, "Monte Carlo filters for non-linear state estimation," Automatica, vol. 37, no. 2, pp. 177-183, Feb. 2001.

[5] S. Boyd, L. E. Ghaoui, E. Feron, and V. Balakrishnan, Linear Matrix Inequalities in System and Control Theory, ser. Studies in Applied Mathematics. Philadelphia, PA: SIAM, 1994.

[6] Y.-Y. Cao and P. M. Frank, "Robust $H_{\infty}$ disturbance attenuation for a class of uncertain discrete-time fuzzy systems," IEEE Trans. Fuzzy Syst., vol. 8, no. 4, pp. 406-415, Aug. 2000.

[7] P. L. Combettes, "The foundations of set-theoretic estimation," Proc. IEEE, vol. 81, no. 2, pp. 182-208, Feb. 1993.

[8] F. Delmotte, T. M. Guerra, and A. Kruszewski, "Discrete Takagi-Sugeno's fuzzy models: Reduction of the number of LMI in fuzzy control techniques," IEEE Trans. Syst., Man, Cybern. B, Cybern., vol. 38, no. 5, pp. 1423-1427, Oct. 2008.

[9] C. Durieu, E. Walter, and B. Polyak, "Multi-input multi-output ellipsoidal state bounding," J. Optim. Theory Appl., vol. 111, no. 2, pp. 273-303, Nov. 2001

[10] H. Gao, J. Lam, L. Xie, and C. Wang, "New approach to mixed $\mathrm{H}_{2} / \mathrm{H}_{\infty}$ filtering for polytopic discrete-time systems," IEEE Trans. Signal Process., vol. 53, no. 8, pp. 3183-3192, Aug. 2005.

[11] Z. Gao, X. Shi, and S. X. Ding, "Fuzzy state/disturbance observer design for T-S fuzzy systems with application to sensor fault estimation," IEEE Trans. Syst., Man, Cybern. B, Cybern., vol. 38, no. 3, pp. 875-880, Jun. 2008.

[12] J. D. Geeter, H. V. Brussel, and J. De Schutter, "A smoothly constrained Kalman filter," IEEE Trans. Pattern Anal. Mach. Intell., vol. 19, no. 10, pp. 1171-1177, Oct. 1997.

[13] L. El Ghaoui and G. Calafiore, "Robust filtering for discrete-time systems with bounded noise and parametric uncertainty," IEEE Trans. Autom. Control, vol. 46, no. 7, pp. 1084-1089, Jul. 2001.

[14] A. B. Kurzhanski and I. Valyi, Ellipsoidal Calculus for Estimation and Control. Boston, MA: Birkhäuser, 1996.

[15] C. Lin, Q.-G. Wang, and T. H. Lee, " $H_{\infty}$ output tracking control for nonlinear systems via T-S fuzzy model approach," IEEE Trans. Syst., Man, Cybern. B, Cybern., vol. 36, no. 2, pp. 450-457, Apr. 2006.

[16] L. Ljung, "Asymptotic behavior of the extended Kalman filter as a parameter estimator for linear systems," IEEE Trans. Autom. Control, vol. AC-24, no. 1, pp. 36-50, Jan. 1979.

[17] J. Löfberg, "YALMIP: A toolbox for modeling and optimization in Matlab," in Proc. IEEE CACSD Symp., Taipei, Taiwan, 2004, pp. 284-289.

[18] D. G. Maskarov and J. P. Norton, "State bounding with ellipsoidal set description of the uncertainty," Int. J. Control, vol. 65, no. 5, pp. 847866, 1996.

[19] D. R. Morrell and W. C. Stirling, "Set-valued filtering and smoothing," IEEE Trans. Syst., Man, Cybern., vol. 21, no. 1, pp. 184-193, Jan. 1991.

[20] D. R. Morrell and W. C. Stirling, "An extended set-valued Kalman filter," in Proc. ISIPTA, 2003, pp. 396-407.

[21] Y. Nesterov and A. Nemirovski, Interior Point Polynomial Methods in Convex Programming: Theory and Applications. Philadelphia, PA: SIAM, 1994.

[22] B. Polyak, S. Nazin, C. Durieu, and E. Walter, "Ellipsoidal estimation under model uncertainty," in 15th Triennial World Congr., Barcelona, Spain, Jul. 2002, pp. 1090-1095.

[23] W. S. Ra, S. H. Jin, and J. B. Park, "Set-valued estimation approach to recursive robust $H_{\infty}$ filtering," Proc. Inst. Elect. Eng.-Control Theory Appl., vol. 151, no. 6, pp. 773-782, Jun. 2004.

[24] K. Reif and R. Unbehauen, "The extended Kalman filter as an exponential observer for nonlinear systems," IEEE Trans. Signal Process., vol. 47, no. 8, pp. 2324-2328, Aug. 1999.

[25] A. V. Savkin and I. R. Petersen, "Robust state estimation and model validation for discrete-time uncertain systems with a deterministic description of noise and uncertainty," Automatica, vol. 34, no. 2, pp. 271-274, Feb. 1998.

[26] E. Scholte and M. E. Campbell, "A nonlinear set-membership filter for on-line applications," Int. J. Robust Nonlinear Control, vol. 13, no. 15, pp. 1337-1358, Dec. 2003

[27] F. C. Schweppe, "Recursive state estimation: Unknown but bounded errors and system inputs," IEEE Trans. Autom. Control, vol. AC-13, no. 1, pp. 22-28, Feb. 1968.

[28] J. S. Shamma and K.-Y. Tu, "Set-valued observers and optimal disturbance rejection," IEEE Trans. Autom. Control, vol. 44, no. 2, pp. 253-264, Feb. 1999.

[29] R. E. Skelton, T. Iwasaki, and K. Grigoriadis, A Unified Algebraic Approach to Linear Control Design. Bristol, PA: Taylor \& Francis, 1998. 
[30] T. Takagi and M. Sugeno, "Fuzzy identification of systems and its applications to modeling and control," IEEE Trans. Syst., Man, Cybern., vol. SMC-15, no. 1, pp. 116-132, Jan. 1985.

[31] K. Tanaka and H. O. Wang, Fuzzy Control Systems Design and Analysis: A Linear Matrix Inequality Approach. New York: Wiley, 2001.

[32] H. Tanizaki, Nonlinear Filters: Estimation and Applications. Berlin, Germany: Springer-Verlag, 1996.

[33] C.-S. Tseng, "Robust fuzzy filter design for nonlinear systems with persistent bounded disturbances," IEEE Trans. Syst., Man, Cybern. B, Cybern., vol. 36, no. 4, pp. 940-945, Aug. 2006.

[34] C. S. Tseng and B. S. Chen, " $H_{\infty}$ fuzzy estimation for a class of nonlinear discrete-time dynamic systems," IEEE Trans. Signal Process., vol. 49, no. 11, pp. 2605-2619, Nov. 2001.

[35] L. Vandenberghe and S. Boyd, "Semidefinite programming," SIAM Rev., vol. 38, no. 1, pp. 49-95, Mar. 1996.

[36] E. A. Wan and R. V. D. Merwe, "The unscented Kalman filter for nonlinear estimation," in Proc. IEEE Symp. Adapt. Syst. Signal Process., Commun., Control, Oct. 2000, pp. 153-158.

[37] H. O. Wang, K. Tanaka, and M. F. Griffin, "An approach to fuzzy control of nonlinear systems: Stability and design issues," IEEE Trans. Fuzzy Syst., vol. 4, no. 1, pp. 14-23, Feb. 1996.

[38] H. S. Witsenhausen, "Sets of possible states of linear systems given perturbed observations," IEEE Trans. Autom. Control, vol. AC-13, no. 5, pp. 556-558, May 1968.

[39] F. Yang, Z. Wang, D. W. C. Ho, and X. Liu, "Robust $H_{2}$ filtering for a class of systems with stochastic nonlinearities," IEEE Trans. Circuits Syst. II, Exp. Briefs, vol. 53, no. 3, pp. 235-239, Mar. 2006.

[40] F. Yang, Z. Wang, Y. S. Hung, and H. Shu, "Mixed $H_{2} / H_{\infty}$ filtering for uncertain systems with regional pole assignment," IEEE Trans. Aerosp. Electron. Syst., vol. 41, no. 2, pp. 438-448, Feb. 2005.

[41] F. Yang, Z. Wang, and Y. S. Hung, "Robust Kalman filtering for discrete time-varying uncertain systems with multiplicative noise," IEEE Trans. Autom. Control, vol. 47, no. 7, pp. 1179-1183, Jul. 2002.

[42] X.-J. Zeng, J. A. Keane, and D. Wang, "Fuzzy systems approach to approximation and stabilization of conventional affine nonlinear systems," in Proc. IEEE Int. Conf. Fuzzy Syst., Vancouver, BC, Canada, Jul. 2006, pp. 277-284.

[43] X.-J. Zeng and M. G. Singh, "Approximation theory of fuzzy systems-MIMO case," IEEE Trans. Fuzzy Syst., vol. 3, no. 2, pp. 219235, May 1995.

[44] H. Gao, Z. Wang, and C. Wang, "Improved H-infinity control of discretetime fuzzy systems: A cone complementarity linearization approach," Inf. Sci., vol. 175 , no. $1 / 2$, pp. 57-77, Sep. 2005.

[45] Z. Wang and B. Huang, "Robust H-2/H-infinity filtering for linear systems with error variance constraints," IEEE Trans. Signal Process., vol. 48, no. 8, pp. 2463-2467, Aug. 2000.

[46] Z. Wang, D. W. C. Ho, and X. Liu, "A note on the robust stability of uncertain stochastic fuzzy systems with time-delays," IEEE Trans. Syst., Man, Cybern. A, Syst., Humans, vol. 34, no. 4, pp. 570-576, Jul. 2004.

[47] B. O. S. Teixeira, J. Chandrasekar, H. J. Palanthandalam-Madapusi, L. A. B. Torres, L. A. Aguirre, and D. S. Bernstein, "Gain-constrained Kalman filtering for linear and nonlinear systems," IEEE Trans. Signal Process., vol. 56, no. 9, pp. 4113-4123, Sep. 2008.

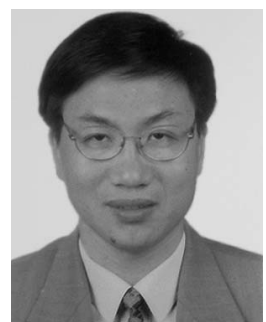

Fuwen Yang (SM'04) received the B.Sc. degree in electrical engineering from Anhui University of Science and Technology, Huainan, China, and the M.Sc. and Ph.D. degrees in control engineering from Huazhong University of Science and Technology, Wuhan, China.

He was previously with Fuzhou University, Fuzhou, China; King's College London, London, U.K.; Brunel University, Middlesex, U.K.; the University of Manchester, Manchester, U.K.; and the University of Hong Kong, Hong Kong. He is currently a Professor with the School of Information Science and Engineering, East China University of Science and Technology, Shanghai, China. He has published more than 100 journal and conference papers in systems control and signal processing. His research interests include $\mathrm{H}$-infinity control and filtering, networked control, fault detection and diagnosis, signal processing, industrial real-time control, and power electronics.

Dr. Yang was the recipient of the 1995 Teaching Excellence Award for Young Teachers from Fok Ying Tung Education Foundation, China; four Science and Technology Development Awards in 1996, 1999, 2002, and 2006, from Fujian Province of China; and two Youth Science and Technology Awards both in 1998 from Fujian Province of China and from Yun Sheng Foundation. He was listed in the Ten Outstanding Youth from Fujian Province of China in 1999 and was honored the May 4 Youth Medal from Fujian Province of China in 2000. Since 2001, he has been acting as a State Consultant receiving Special Allowance from the State Council of the People's Republic of China.

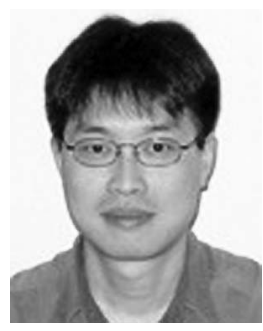

Yongmin $\mathbf{L i}$ (SM'06) received the B.Eng. and M.Eng. degrees in control engineering from Tsinghua University of China, Beijing, China, in 1990 and 1992, respectively, and the Ph.D. degree in computer vision from Queen Mary, University of London, London, U.K., in 2001.

He was a Research Scientist with the British Telecom Laboratories. He is currently a faculty member with the Department of Information Systems and Computing, Brunel University, Middlesex, U.K. His research interest covers the areas of automatic control, machine learning, pattern recognition, computer vision, image processing, video analysis, medical imaging, and bioimaging.

Dr. Li received the British Machine Vision Association (BMVA) Best Scientific Award in 2001 and the Best Paper Prize from IEEE International Workshop on Recognition, Analysis and Tracking of Faces and Gestures (RATFG) in 2001 . 